\title{
A note on irreducible maps with several boundaries
}

\author{
J. Bouttier ${ }^{1,2} \quad$ E. Guitter ${ }^{1}$ \\ ${ }^{1}$ Institut de Physique Théorique \\ CEA, IPhT, F-91191 Gif-sur-Yvette, France \\ CNRS, URA 2306 \\ 2 Département de Mathématiques et Applications \\ École normale supérieure, 45 rue d'Ulm, F-75231 Paris Cedex 05 \\ \{jeremie.bouttier, emmanuel.guitter\}@cea.fr
}

Submitted: Jun 7, 2013; Accepted: Jan 23, 2014; Published: Feb 7, 2014

\begin{abstract}
We derive a formula for the generating function of $d$-irreducible bipartite planar maps with several boundaries, i.e. having several marked faces of controlled degrees. It extends a formula due to Collet and Fusy for the case of arbitrary (non necessarily irreducible) bipartite planar maps, which is recovered by taking $d=0$. As an application, we obtain an expression for the number of $d$-irreducible bipartite planar maps with a prescribed number of faces of each allowed degree. Very explicit expressions are given in the case of maps without multiple edges $(d=2)$, 4 -irreducible maps and maps of girth at least $6(d=4)$. Our derivation is based on a tree interpretation of the various encountered generating functions.
\end{abstract}

\section{Introduction}

This note is an extension of a preceding paper [1] on the enumeration of $d$-irreducible planar maps via slice decomposition. We shall use here a number of results from this paper, which the reader is invited to consult for explicit proofs.

We recall that a planar map is a cellular embedding of a graph in the sphere, considered up to continuous deformation. A map is bipartite if one can color its vertices in black and white so that no two adjacent vertices have the same color. A necessary and sufficient condition for a planar map to be bipartite is that all its faces have even degrees. This paper deals exclusively with planar bipartite maps.

Ref. [1] was mainly devoted to the enumeration of maps with a single boundary, i.e. maps with a distinguished oriented edge (the root edge) and with a control on its outer degree, i.e. the degree of the face lying on the right of this edge (the root face). By first choosing the root face, then the root edge, maps with a single boundary may alternatively 
be defined as maps with a distinguished face of controlled degree and with a marked oriented edge incident to this face whose orientation is such that the distinguished face lies on its right. In this paper, we shall extend this definition to maps with a number $r \geqslant 2$ of boundaries, namely maps with $r$ distinguished faces of controlled degrees, and with a marked oriented edge incident to each distinguished face (again with an orientation such that the distinguished face at hand lies on its right). The faces which have not been distinguished will be referred to as inner faces. Note that maps with $r \geqslant 2$ boundaries have at least two faces, so they cannot be reduced to trees. The case of maps with two boundaries was actually discussed already at the end of Ref. [1] and we shall rely here on the corresponding results.

Throughout this paper, we consider $d$ some even integer larger than or equal to 2 . We set for convenience

$$
d=2 b
$$

with $b$ an integer larger than or equal to 1 . The results presented here concern the enumeration of so-called d-irreducible maps, as defined now. Recall that the girth of a map is the minimal length (number of edges) of its cycles (simple closed paths). Note that the girth of a bipartite map is necessarily even. A map with one or several boundaries is said $d$-irreducible if its girth is at least $d$ and if all its cycles of length $d$ are the boundary of an inner face of degree $d$. Clearly, in a $d$-irreducible map with $r \geqslant 2$ boundaries, all inner faces have a degree larger than or equal to $d$ (by the girth condition) and all the boundary faces have a degree strictly larger than $d$ (by our definition, a $d$-irreducible map with a boundary face of degree $d$ is either reduced to a tree or has a single inner face of degree $d$ and no other boundary face). Thus, the only non-trivial case to consider is when all the marked faces have a degree strictly larger than $d$. We shall enumerate $d$-irreducible maps with a weight $z$ per $d$-valent inner face and a weight $x_{2 j}$ per $2 j$-valent inner face for $j>b$ (recall that all faces have even degree in a bipartite map), the marked faces receiving no weight. We then denote by

$$
F_{2 j_{1}, 2 j_{2}, \ldots, 2 j_{r}}^{(d)} \equiv F_{2 j_{1}, 2 j_{2}, \ldots, 2 j_{r}}^{(d)}\left(z ; x_{d+2}, x_{d+4}, \ldots\right)
$$

the generating function of $d$-irreducible maps with $r$ boundaries whose marked faces have respective degrees $2 j_{1}, 2 j_{2}, \ldots, 2 j_{r}$, with $j_{\ell} \geqslant b, \ell=1, \ldots r$ (it vanishes as soon as $j_{\ell}=b$ for some $\ell$ ). The main result of this paper is a general expression for this generating function:

Theorem 1. For $d=2 b \geqslant 2$ and $r \geqslant 2$, we have

$$
F_{2 j_{1}, 2 j_{2}, \ldots, 2 j_{r}}^{(d)}=\frac{1}{(r-2) b+\sum_{\ell=1}^{r} j_{\ell}} \prod_{\ell=1}^{r} 2 j_{\ell}\left(\begin{array}{c}
2 j_{\ell}-1 \\
j_{\ell}+b
\end{array}\right) \frac{\partial^{r-2}}{\partial z^{r-2}}\left(R^{(d)}\right)^{(r-2) b+\sum_{\ell=1}^{r} j_{\ell}}
$$

where $R^{(d)}$ is the formal power series determined by

$$
z+\sum_{\ell=0}^{b}(-1)^{b-\ell}\left(\begin{array}{c}
b+\ell \\
2 \ell
\end{array}\right) \operatorname{Cat}(\ell)\left(R^{(d)}\right)^{b-\ell}+\sum_{j \geqslant b+1}\left(\begin{array}{c}
2 j-1 \\
j+b
\end{array}\right) x_{2 j}\left(R^{(d)}\right)^{b+j}=0 .
$$


In particular, if we impose an upper bound on the degree of the faces (i.e. $x_{2 j}$ vanishes for $j$ large enough), then $F_{2 j_{1}, 2 j_{2}, \ldots, 2 j_{r}}^{(d)}$ is algebraic.

Note that, if we take formally $d=0$ (i.e. $b=0$ ) in $(1.3)$, we recover a formula due to Collet and Fusy [2] for the case of arbitrary (non necessarily irreducible) bipartite planar maps, upon understanding $z$ as a weight per vertex and identifying $R^{(0)}$ properly (beware that (1.4) is not valid for $d=0$ ), see the discussion in Section 4 below.

The paper is organized as follows. Section 2 presents a proof of Theorem 1. Our starting point is a formula from [1] for the generating function of $d$-irreducible maps with $r=2$ boundaries, which we recall in Section 2.1. We then show in Section 2.2 how to interpret the various intermediate functions at hand as generating functions for decorated trees. The core of the proof of Theorem 1 comes in Section 2.3: the generating function of maps with several boundaries is shown to coincide with that of trees with several marked vertices, which are themselves, via a construction reminiscent of that of [2], in bijection with appropriate forests. Section 3 discusses applications to enumeration, i.e. expressions for numbers of rooted bipartite planar $d$-irreducible maps with a prescribed face degree distribution. A general formula is given in Section 3.1. By taking $d=2$ and forbidding inner bivalent faces, we recover in Section 3.2 a formula from [4] concerning maps without multiple edges. The cases of 4 -irreducible maps and maps of girth at least 6 are discussed very explicitly in the respective Sections 3.3 and 3.4. Section 4 shows how the Collet-Fusy formula is recovered by taking formally $d=0$ in our formulas. A few concluding remarks are gathered in Section 5.

\section{Generating functions for $d$-irreducible maps}

\subsection{The starting point: $d$-irreducible maps with two boundaries}

Our starting point is a formula for the quantity (1.2) at $r=2$, namely the generating function $F_{2 j_{1}, 2 j_{2}}^{(d)}$ of $d$-irreducible bipartite maps with two boundaries of respective lengths $2 j_{1}$ and $2 j_{2}$. From [1, eq. (9.17)], it is given by

$$
F_{2 j_{1}, 2 j_{2}}^{(d)}=2 j_{1}\left(\begin{array}{c}
2 j_{1}-1 \\
j_{1}+b
\end{array}\right) 2 j_{2}\left(\begin{array}{c}
2 j_{2}-1 \\
j_{2}+b
\end{array}\right) \frac{\left(R^{(d)}\right)^{j_{1}+j_{2}}}{j_{1}+j_{2}} \quad j_{1}, j_{2}>b
$$

where the function $R^{(d)}$ is a fundamental object related via

$$
R^{(d)}=1+U_{0}^{(d)}
$$

to the first member $U_{0}^{(d)}$ of a family of slice generating functions $U_{k}^{(d)}, 0 \leqslant k \leqslant b$. We shall not explain here what are precisely the "slices" enumerated by $U_{k}^{(d)}$ and refer to [1] for such a discussion. We remind simply that slices appear as the result of a cutting of the maps along geodesic paths, and have themselves a recursive decomposition. As 


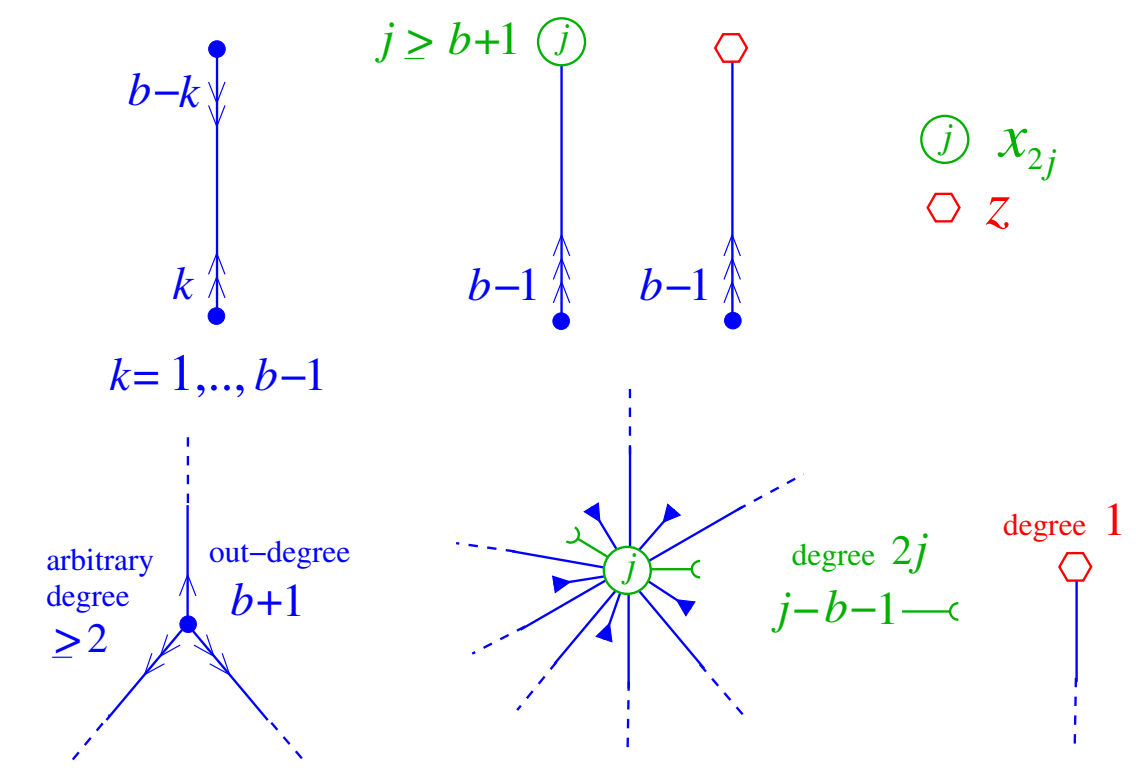

Figure 1: Tree building rules in the case $b>1$ (see text).

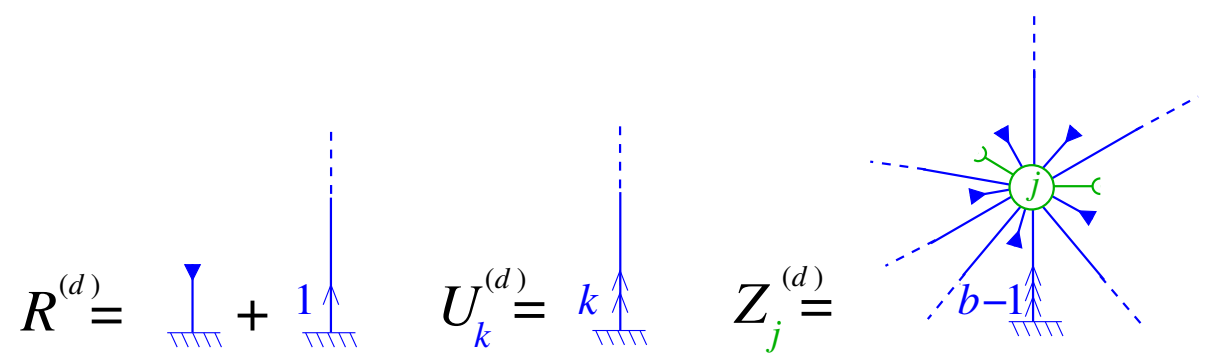

Figure 2: Interpretation of $R^{(d)}, U_{k}^{(d)}, 1 \leqslant k \leqslant b-1$ and $Z_{j}^{(d)}, j \geqslant b+1$ as generating functions for planted trees.

a consequence, the generating functions $U_{k}^{(d)}$ are fully determined via a set of recursive equations

$$
U_{k}^{(d)}=z \delta_{k, b-1}+\sum_{q \geqslant 1} \sum_{\substack{k_{1}, \ldots, k_{q} \geqslant 1 \\ k_{1}+\cdots+k_{q}=k+1}} \prod_{i=1}^{q} U_{k_{i}}^{(d)} \quad 0 \leqslant k \leqslant b-1,
$$

which, together with (2.2), form a closed system, once completed by the relation

$$
U_{b}^{(d)}=\sum_{j \geqslant b+1}\left(\begin{array}{c}
2 j-1 \\
j+b
\end{array}\right) x_{2 j}\left(R^{(d)}\right)^{j+b} .
$$

Our goal is to extend formula (2.1) to the case of maps with $r>2$ boundaries. 


\subsection{The tree representation}

For $b>1$, we may eliminate explicitly $U_{0}^{(d)}$ and $U_{b}^{(d)}$ from the system (2.2)-(2.4) by using $U_{0}^{(d)}=U_{1}^{(d)}$ (as obtained from (2.3) at $k=0$ ) and using the expression (2.4) for $U_{b}^{(d)}$, resulting in the system

$$
\begin{aligned}
R^{(d)} & =1+U_{1}^{(d)} \\
U_{k}^{(d)} & =\sum_{q \geqslant 1} \sum_{\substack{1 \leqslant k_{1}, \ldots, k_{q} \leqslant b-1 \\
k_{1}+\cdots+k_{q}=k+1}} \prod_{i=1}^{q} U_{k_{i}}^{(d)} \quad 1 \leqslant k \leqslant b-2 \\
U_{b-1}^{(d)} & =\sum_{\substack { q \geqslant 2 \\
\begin{subarray}{c}{1 \leqslant k_{1}, \ldots, k_{q} \leqslant b-1 \\
k_{1}+\cdots+k_{q}=b{ q \geqslant 2 \\
\begin{subarray} { c } { 1 \leqslant k _ { 1 } , \ldots , k _ { q } \leqslant b - 1 \\
k _ { 1 } + \cdots + k _ { q } = b } }\end{subarray}} \prod_{i=1}^{q} U_{k_{i}}^{(d)}+\left(z+\sum_{j \geqslant b+1} Z_{j}^{(d)}\right) \\
Z_{j}^{(d)} & =\left(\begin{array}{c}
2 j-1 \\
j+b
\end{array}\right) x_{2 j}\left(R^{(d)}\right)^{j+b} \quad j \geqslant b+1 .
\end{aligned}
$$

In view of this system, we may reinterpret $R^{(d)}, U_{k}^{(d)}, 1 \leqslant k \leqslant b-1$ and $Z_{j}^{(d)}, j \geqslant b+1$ as generating functions for particular planted plane trees built according to the following rules, displayed in fig. 1 . The trees are made of

- four types of vertices:

- black inner vertices (represented by filled dots);

- white inner vertices carrying an arbitrary integer label $j \geqslant b+1$ (represented as circles with the label $j$ inside);

- leaf-vertices (represented by hexagons);

- a univalent root-vertex (represented by the ground symbol in fig. 2);

- two types of edges:

- bi-oriented edges, i.e. edges carrying an arbitrary number $k$ between 1 and $b-1$ of outgoing arrows pointing away from one of their extremities, and $b-k$ outgoing arrows pointing away from the other extremity. These bi-oriented edges connect only black inner vertices or the root;

- mono-oriented edges, carrying $b-1$ outgoing arrows pointing away from one of their extremities, being necessarily a black inner vertex or the root. The other extremity (without arrows) is necessarily a white inner vertex or a leaf-vertex;

- with the vertex constraints:

- black inner vertices have arbitrary degrees larger than or equal to 2 but a fixed out-degree equal to $b+1$. By out-degree of a vertex, we mean the total number of outgoing arrows pointing away from it. (Note that the out-degree constraint restricts in practice the degree of black inner vertices, which can be at most $b+1)$; 


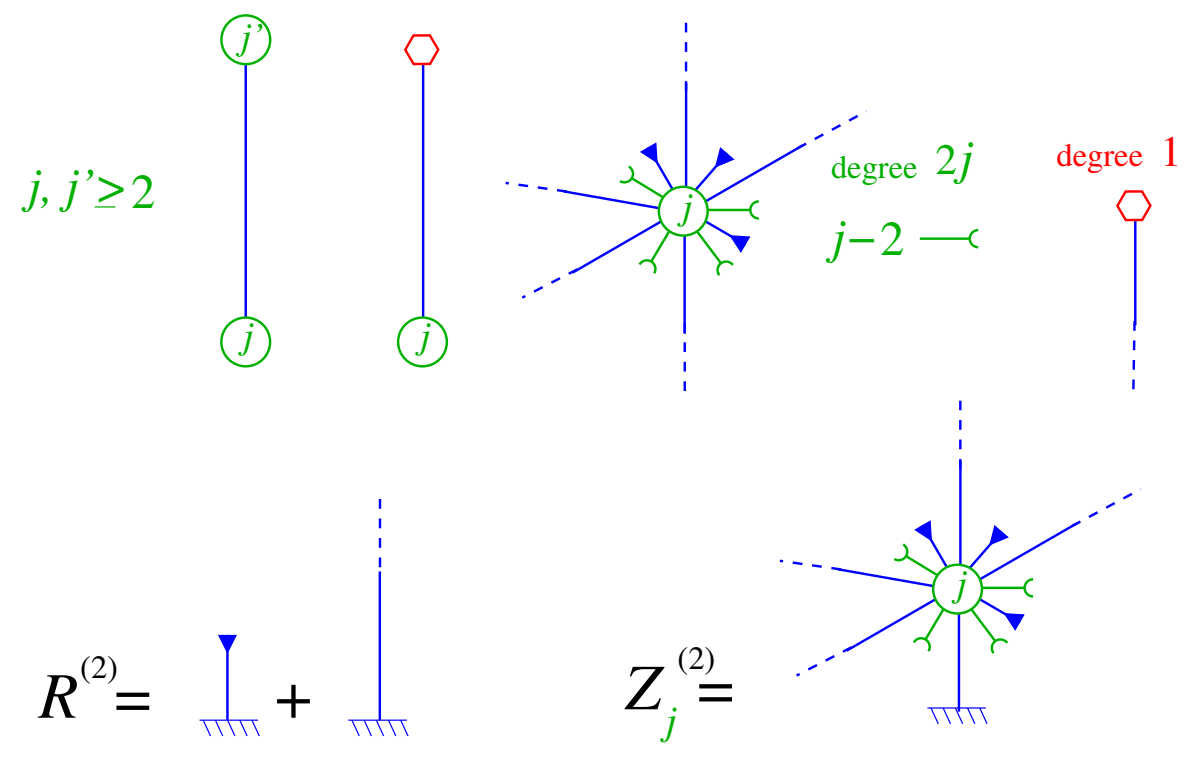

Figure 3: Tree building rules in the case $b=1$.

- white inner vertices are incident to mono-oriented edges and to two types of decorations: buds (represented with a semi-circular endpoint) and blossoms (represented with a triangular endpoint). A white inner vertex with label $j$ has total degree $2 j$ (including blossoms and buds) and a number $j-b-1$ of buds. It is weighted by $x_{2 j}$;

- leaf-vertices have degree 1 and are weighted by $z$.

The reader will easily check that the rules above are designed so as to reproduce the equations (2.5) by a canonical decomposition of the (supposedly planted) trees into descending subtrees by cutting them at the level of their first vertex. This vertex can be a black inner vertex of degree $q+1(q \geqslant 1)$, reproducing the $q$-dependent terms in $(2.5)$, a white inner vertex with label $j$, reproducing the $j$-dependent terms in (2.5), or a leaf-vertex, reproducing the $z$-dependent term in (2.5). More precisely, $R^{(d)}, U_{k}^{(d)}, 1 \leqslant k \leqslant b-1$ and $Z_{j}^{(d)}, j \geqslant b+1$ are easily identified with the generating functions for the planted trees displayed in fig. 2. Let us mention that similar trees (without leaf-vertices) were introduced in $[3,4]$ in the context of maps with controlled girth. This is consistent with the fact that, as observed in [1], enumerating such maps indeed corresponds to imposing $z=0$ in our setting.

For $b=1$, eqs. (2.2)-(2.4) simply reduce to

$$
\begin{aligned}
& R^{(2)}=1+z+\sum_{j \geqslant 2} Z_{j}^{(2)} \\
& Z_{j}^{(2)}=\left(\begin{array}{c}
2 j-1 \\
j+1
\end{array}\right) x_{2 j}\left(R^{(2)}\right)^{j+1}, \quad j \geqslant 2
\end{aligned}
$$




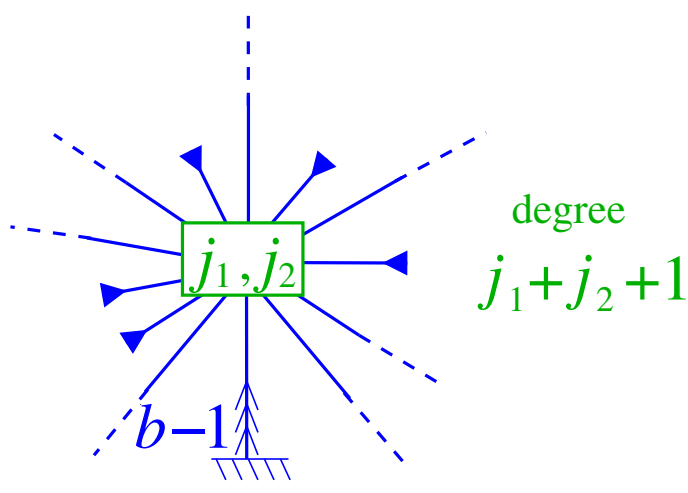

Figure 4: The building rule of the special vertex labeled by $j_{1}$ and $j_{2}$. It has degree $j_{1}+j_{2}+1$ and is incident to mono-oriented (or un-oriented if $b=1$ ) edges or blossoms only. The edge connecting it to the root is a mono-oriented edge (with thus $b-1$ arrows pointing away from the root - it is un-oriented if $b=1$ ).

and may be viewed as generating the simpler trees of fig. 3 with un-oriented edges.

\subsection{A formula for the generating function of $d$-irreducible maps with $r \geqslant 2$ boundaries}

Returning to the general case $b \geqslant 1$, we now consider the generating function $F_{2 j_{1}, 2 j_{2}, \ldots, 2 j_{r}}^{(d)}$ for maps with $r \geqslant 2$ boundaries. Since $r \geqslant 2$, maps enumerated by $F_{2 j_{1}, 2 j_{2}, \ldots, 2 j_{r}}^{(d)}$ may be obtained from maps enumerated by $F_{2 j_{1}, 2 j_{2}}^{(d)}$ by a simple marking of $r-2$ extra faces of respective degrees $2 j_{3}, 2 j_{4}, \ldots 2 j_{r}$. After marking, these extra faces are no longer considered as inner faces. Still the constraint that $d$-cycles must be boundaries of inner faces of degree $d$ is not affected by the marking upon assuming that the marked faces all have degree strictly larger than $d$ (i.e. we assume from now on $j_{\ell}>d, \ell=1, \ldots, r$ ). Note that Theorem 1 is trivially true when this assumption does not hold, for both sides of (1.3) vanish. At the level of generating function, the marking is performed via the action of derivatives with respect to $x_{2 j_{\ell}}$ for $\ell=3, \ldots r$, namely

$$
F_{2 j_{1}, 2 j_{2}, \ldots, 2 j_{r}}^{(d)}=\left(\prod_{\ell=3}^{r} 2 j_{\ell} \frac{\partial}{\partial x_{2 j_{\ell}}}\right) F_{2 j_{1}, 2 j_{2}}^{(d)}, \quad r \geqslant 2
$$

(with the usual convention that the empty product represents the identity operator). Here the $2 j_{\ell}$ factors account for the choice of an oriented edge incident to each newly marked face.

From (2.1), we may thus write

$$
F_{2 j_{1}, 2 j_{2}, \ldots, 2 j_{r}}^{(d)}=\prod_{\ell=1}^{r} 2 j_{\ell} \times \frac{\left(\begin{array}{c}
2 j_{1}-1 \\
j_{1}+b
\end{array}\right)\left(\begin{array}{c}
2 j_{2}-1 \\
j_{2}+b
\end{array}\right)}{j_{1}+j_{2}} \times H_{2 j_{1}, 2 j_{2} ; 2 j_{3}, \ldots, 2 j_{r}}^{(d)}
$$




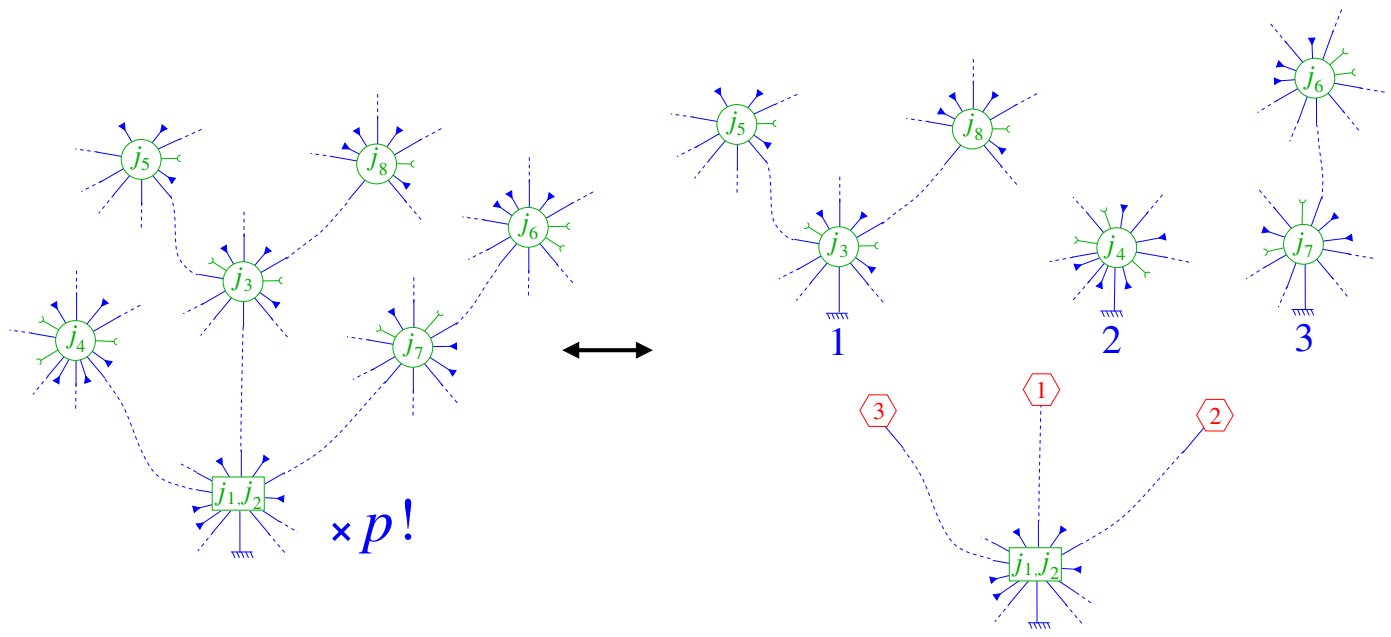

Figure 5: Schematic picture of the bijection between the trees enumerated by $H_{2 j_{1}, 2 j_{2} ; 2 j_{3}, \ldots, 2 j_{r}}$ with a number $p$ of first generation vertices, supposedly numbered 1 to $p$ is all possible ways (hence the factor $p$ !) and pairs made of (i) a forest of $p$ trees with roots labeled 1 to $p$, with $(r-p-2)$ marked non-root white vertices and (ii) a tree with first special vertex and $p$ marked leaf-vertices labeled 1 to $p$. Here $p=3$ and $r=8$.

where

$$
H_{2 j_{1}, 2 j_{2} ; 2 j_{3}, \ldots, 2 j_{r}}^{(d)}=\left(\prod_{\ell=3}^{r} \frac{\partial}{\partial x_{2 j_{\ell}}}\right)\left(R^{(d)}\right)^{j_{1}+j_{2}}
$$

In the tree language, the function $H_{2 j_{1}, 2 j_{2} ; 2 j_{3}, \ldots, 2 j_{r}}^{(d)}$ can be interpreted as the generating function for planted trees built according to the rules of fig. 1 (or of fig. 3 if $b=1$ ), whose root is adjacent to a first special vertex carrying both labels $j_{1}$ and $j_{2}$, as displayed in fig. 4 , and with $r-2$ marked white inner vertices of respective degrees $2 j_{3}, 2 j_{3}, \ldots, 2 j_{r}$. The building rule of the special vertex is designed to reproduce the term $\left(R^{(d)}\right)^{j_{1}+j_{2}}$ in (2.9): this special vertex must have total degree $j_{1}+j_{2}+1$ and be incident to monooriented (or un-oriented if $b=1$ ) edges or blossoms only. The special vertex and the $r-2$ marked white inner vertices receive no weight. Let us now show that we may write the generating function $H_{2 j_{1}, 2 j_{2} ; 2 j_{3}, \ldots, 2 j_{r}}^{(d)}$ as

$$
\begin{aligned}
H_{2 j_{1}, 2 j_{2} ; 2 j_{3}, \ldots, 2 j_{r}}^{(d)} & =\prod_{\ell=3}^{r}\left(\begin{array}{c}
2 j_{\ell}-1 \\
j_{\ell}+b
\end{array}\right) \\
& \times \sum_{p=1}^{r-2}\left(\begin{array}{c}
r-3 \\
p-1
\end{array}\right) \frac{\partial^{p}}{\partial z^{p}}\left(R^{(d)}\right)^{j_{1}+j_{2}} \frac{\partial^{r-p-2}}{\partial z^{r-p-2}}\left(R^{(d)}\right)^{\sum_{\ell=3}^{r}\left(j_{\ell}+b\right)} .
\end{aligned}
$$

We shall proceed in two steps: the trees enumerated by $H_{2 j_{1}, 2 j_{2} ; 2 j_{3}, \ldots, 2 j_{r}}^{(d)}$ have a number $p \geqslant 1$ of "first generation" marked white vertices, which have the special vertex as "direct" ancestor, i.e. are such that the branch from the special vertex to them does not pass via 

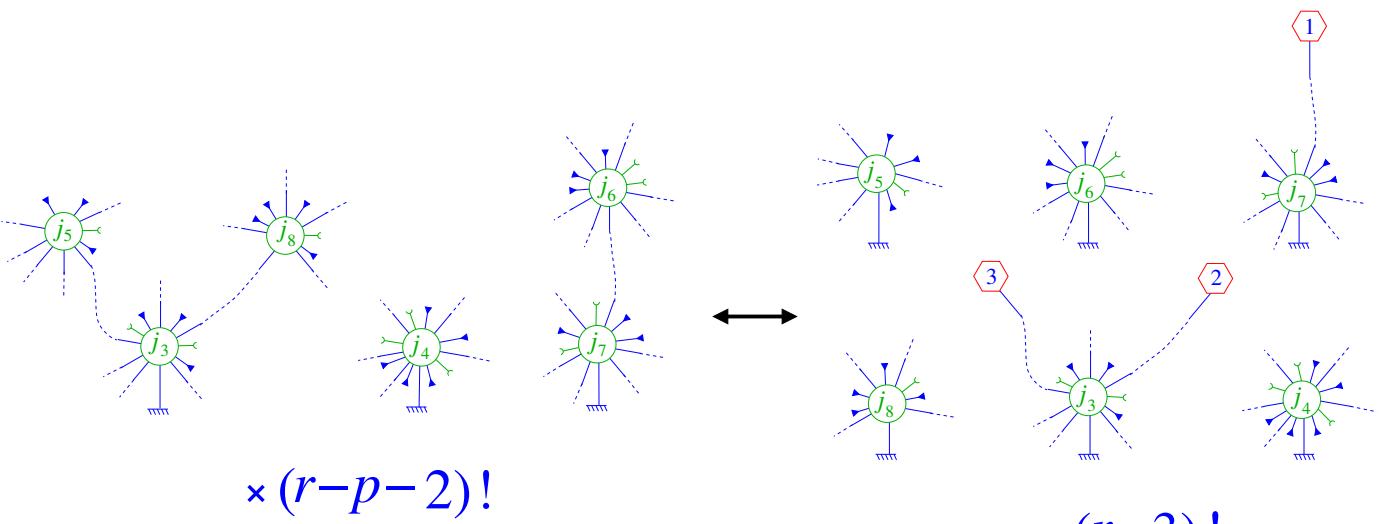

$\times \frac{(r-3) !}{(p-1) !}$

Figure 6: Schematic picture of the correspondence between forests of $p$ unlabeled trees with $(r-p-2)$ marked non-root white vertices and forests with $(r-2)$ unlabeled trees equipped with a total of $(r-p-2)$ marked leaf-vertices labeled 1 to $(r-p-2)$. The correspondence is $(r-p-2)$ ! to $(r-3) ! /(p-1)$ ! (see text). Here $p=3$ and $r=8$.

any other marked white vertex. We number these first generation vertices from 1 to $p$ (in all possible ways so that each tree is counted $p$ ! times - see fig. 5 , left) and cut the tree at the level of their mono-oriented incident edge in the ascendent part of the tree. The $p$ cut parts form a forest made of $p$ rooted trees, whose roots are incident to the $p$ first generation vertices, and are labeled 1 to $p$ (see fig. 5, right). This forest is equipped with a total of $(r-p-2)$ marked white vertices (those which are not first generation). As for the part containing the special vertex, we may repair it by adding a leaf-vertex to each of the cut mono-oriented edges, thus re-creating a tree satisfying the rules of fig. 1, with root adjacent to the special vertex, and with now $p$ marked (and labeled) leaf-vertices. These marked leaf-vertices receive no weight, so the generating function for such trees is simply

$$
\frac{\partial^{p}}{\partial z^{p}}\left(R^{(d)}\right)^{j_{1}+j_{2}}
$$

This explains the $j_{1}, j_{2}$-dependent term in (2.10). Moreover, the $p$ ! to 1 correspondence displayed in fig. 5 can be made 1 to 1 by simply removing the labeling of the tree roots in the forest part.

In a second step, the enumeration of the complementary forest part is achieved as in [2] via a (many-to-many) correspondence between forests of $p$ trees with a total of $(r-p-2)$ marked white non-root vertices (left side of fig. 6) and forests of $(r-2)$ trees and a total of $(r-p-2)$ marked leaf-vertices (right side of fig. 6). We proceed as before by first labeling the $(r-p-2)$ marked white non-root vertices in all possible ways (each forest is thus counted $(r-p-2)$ ! times) and cutting the trees in the forest at the level of the mono-oriented edges leading to all these vertices. The cut mono-oriented edges are as before completed by new added leaf-vertices in the ascending part of the trees and serve 
as roots for the descending parts. The net result is a forest of $(r-2)$ trees whose roots are incident to the $(r-2)$ marked white vertices with degrees $2 j_{3}, \ldots, 2 j_{r}$, equipped with a total of $(r-p-2)$ marked leaf-vertices labeled 1 to $(r-p-2)$. These forests are counted by

$$
\frac{\partial^{r-p-2}}{\partial z^{r-p-2}} \prod_{\ell=3}^{r}\left(\left(\begin{array}{c}
2 j_{\ell}-1 \\
j_{\ell}+b
\end{array}\right)\left(R^{(d)}\right)^{j_{\ell}+b}\right)
$$

which explains the $\left(j_{3} \rightarrow j_{r}\right)$-dependent terms in (2.10). The correspondence is not simply $(r-p-2)$ ! to 1 since, in $(2.12)$, we erased the information on how to reassemble the trees. The appropriate counting is more transparent if we start conversely from a forest of $(r-2)$ trees with a total of $(r-p-2)$ marked leaves: we then rebuild a forest of $p$ trees and $(r-p-2)$ marked (and labeled) white non-root vertices by first replacing the marked leaf-vertex labeled 1 by a descending subtree chosen among any of the trees in the forest which do not carry this marked leaf-vertex ( $r-3$ choices), creating a new forest with one less tree, then replacing the marked leaf-vertex labeled 2 by a descending subtree formed by any of the trees in the new forest which do not carry this marked leaf-vertex $(r-4$ choices $)$, and continue until the $(r-p-2)$-th marked leaf-vertex in replaced by a descending subtree ( $p$ choices). This leaves us with the desired forest with $p$ trees. Clearly, each configuration is counted $(r-3) ! /(p-1)$ ! times so that the correspondence is eventually $(r-p-2)$ ! to $(r-3) ! /(p-1)$ !, which explains the binomial factor $\left(\begin{array}{l}r-3 \\ p-1\end{array}\right)$ in (2.10).

Now the sum in (2.10) may be simply evaluated through

$$
\begin{aligned}
& \sum_{p=1}^{r-2}\left(\begin{array}{l}
r-3 \\
p-1
\end{array}\right) \frac{\partial^{p}}{\partial z^{p}}\left(R^{(d)}\right)^{j_{1}+j_{2}} \frac{\partial^{r-p-2}}{\partial z^{r-p-2}}\left(R^{(d)}\right)^{\sum_{\ell=3}^{r}\left(j_{\ell}+b\right)} \\
& =\frac{\partial}{\partial \zeta}\left\{\sum_{p=1}^{r-2}\left(\begin{array}{l}
r-3 \\
p-1
\end{array}\right) \frac{\partial^{p-1}}{\partial z^{p-1}}\left(R^{(d)}(z+\zeta)\right)^{j_{1}+j_{2}} \frac{\partial^{r-p-2}}{\partial z^{r-p-2}}\left(R^{(d)}(z)\right)_{\ell=3}^{\sum_{\ell=3}^{r}\left(j_{\ell}+b\right)}\right\}_{\zeta=0} \\
& =\frac{\partial}{\partial \zeta}\left\{\frac{\partial^{r-3}}{\partial z^{r-3}}\left(\left(R^{(d)}(z+\zeta)\right)^{j_{1}+j_{2}}\left(R^{(d)}(z)\right)^{\sum_{\ell=3}^{r}\left(j_{\ell}+b\right)}\right)\right\}_{\zeta=0} \\
& =\frac{\partial^{r-3}}{\partial z^{r-3}} \frac{\partial}{\partial \zeta}\left\{\left(R^{(d)}(z+\zeta)\right)^{j_{1}+j_{2}}\left(R^{(d)}(z)\right)^{\sum_{\ell=3}^{r}\left(j_{\ell}+b\right)}\right\}_{\zeta=0} \\
& \left.=\frac{\partial^{r-3}}{\partial z^{r-3}} \frac{j_{1}+j_{2}}{j_{1}+j_{2}+\sum_{\ell=3}^{r}\left(j_{\ell}+b\right)}\right\}_{\zeta=0}\left\{\left(R^{(d)}(z+\zeta)\right)^{j_{1}+j_{2}+\sum_{\ell=3}^{r}\left(j_{\ell}+b\right)}\right\}_{j^{\prime}} \\
& =\frac{j_{1}+j_{2}}{(r-2) b+\sum_{\ell=1}^{r} j_{\ell} \frac{\partial^{r-2}}{\partial z^{r-2}}\left(R^{(d)}\right)}(r-2) b+\sum_{\ell=1}^{r} j_{\ell} .
\end{aligned}
$$

Here we simply used Leibniz formula to go from the second to the third line, as well as elementary operations. Plugging this result in (2.10) and (2.8), we arrive at our main 
formula (1.3). In this formula, the function $R^{(d)}$ is the solution of eqs. (2.2)-(2.4). As shown in [1], this system reduces after elimination to the single equation (1.4) for $R^{(d)}$. This completes the proof of Theorem 1.

\section{Enumeration formulas}

\subsection{A general formula}

A direct corollary of Theorem 1 is a formula for the number $N_{m}^{(d)}\left(\left\{q_{j}\right\}_{j \geqslant b}\right)$ of rooted bipartite planar $d$-irreducible maps with outer degree $2 m(m \geqslant b+1)$ and with $q_{j}$ faces of degree $2 j, j \geqslant b$ (distinct from the root face if $j=m$ ). It is obtained by marking in the map all the faces with degree strictly larger than $b$, leaving the $d$-valent faces only as inner faces. In (1.3), the function $R^{(d)}$ must therefore be evaluated at $x_{2 j}=0$ for all $j>b$. We call $r^{(d)}(z)$ this function, which is obtained by solving $(2.5)$ (or $(2.6)$ if $b=1$ ) at $Z_{j}^{(d)}=0$ for all $j$. It is determined by the equation

$$
z+\sum_{\ell=0}^{b}(-1)^{b-\ell}\left(\begin{array}{c}
b+\ell \\
2 \ell
\end{array}\right) \operatorname{Cat}(\ell)\left(r^{(d)}\right)^{b-\ell}=0
$$

as read off eq. (1.4) at $x_{2 j}=0, j>b$. Eq. (1.3) translates directly into:

Proposition 2. For $m$ and $q_{b}, q_{b+1}, q_{b+2}, \ldots$ nonnegative integers such that $m \geqslant b+1$ and $1 \leqslant \sum_{j>b} q_{j}<\infty$, the number $N_{m}^{(d)}\left(\left\{q_{j}\right\}_{j \geqslant b}\right)$ of rooted bipartite planar d-irreducible maps with outer degree $2 m$ and with $q_{j}$ non-root faces of degree $2 j$ for all $j$ reads

$$
\begin{aligned}
N_{m}^{(d)}\left(\left\{q_{j}\right\}_{j \geqslant b}\right)= & (2 m)\left(\begin{array}{c}
2 m-1 \\
m+b
\end{array}\right) \prod_{j>b} \frac{1}{q_{j} !}\left(\begin{array}{c}
2 j-1 \\
j+b
\end{array}\right)^{q_{j}} \\
& \times \frac{1}{E+b\left(F-2-2 q_{b}\right)} \frac{(F-2) !}{q_{b} !}\left[z^{F-2}\right]\left(r^{(d)}(z)\right)^{E+b\left(F-2-2 q_{b}\right)}
\end{aligned}
$$

where $F$ and $E$ are the total numbers of faces and edges respectively, and $r^{(d)}$ is determined by (3.1).

Note the absence of the factors $2 j$ in the product which, in (1.3), accounted for a choice of edge incident to each marked face. Here only the root face receives such a factor. Note also the factorial factors since, as opposed to (1.3), the marked faces are not distinguished. We have used the identities

$$
\begin{aligned}
& F=1+q_{b}+\sum_{j>b} q_{j} \\
& E=m+b q_{b}+\sum_{j>b} j q_{j}
\end{aligned}
$$


which lead to the identifications $r=1+\sum_{j>b} q_{j}=F-q_{b}$ and $(r-2) b+\sum_{\ell=1}^{r} j_{\ell}=(r-2) b+$ $m+\sum_{j>b} j q_{j}=E+b\left(F-2-2 q_{b}\right)$. Expression (3.2) is valid for $F=1+q_{b}+\sum_{j>b} q_{j} \geqslant 2+q_{b}$, i.e. with at least two faces of degree strictly larger than $b$.

\subsection{Bipartite maps without multiple edges}

A first case of interest corresponds to bipartite maps without multiple edges, i.e. bipartite maps of girth at least 4 . These maps are simply obtained from 2-irreducible maps $(b=1)$ by forbidding bivalent faces, i.e. setting $q_{1}=0$. Using $r^{(2)}(z)=1+z$ (as seen directly from $(2.6)$ at $Z_{j}^{(2)}=0$ for all $j$ ), and

$$
(F-2) !\left[z^{F-2}\right](1+z)^{E+F-2}=\frac{(E+F-2) !}{E !}
$$

we obtain from Proposition 2 that:

Proposition 3. For $m \geqslant 2$ and $q_{2}, q_{3}, \ldots$ nonnegative integers, the number $\mathcal{N}_{m}^{(4)}\left(\left\{q_{j}\right\}_{j \geqslant 2}\right)$ $\left(=N_{m}^{(2)}\left(\left\{q_{j}\right\}_{j \geqslant 1}\right)\right.$ at $\left.q_{1}=0\right)$ of rooted bipartite planar maps without multiple edges, with outer degree $2 m$ and with $q_{j}$ non-root faces of degree $2 j$ for all $j$ reads

$$
\mathcal{N}_{m}^{(4)}\left(\left\{q_{j}\right\}_{j \geqslant 2}\right)=(2 m)\left(\begin{array}{c}
2 m-1 \\
m+1
\end{array}\right) \prod_{j>1} \frac{1}{q_{j} !}\left(\begin{array}{c}
2 j-1 \\
j+1
\end{array}\right)^{q_{j}} \frac{(E+F-3) !}{E !}
$$

where $E=m+\sum_{j>1} j q_{j}$ is the total number of edges, and $F=1+\sum_{j>1} q_{j}$ the total number of faces.

This expression agrees with Proposition 35 in [4], up to a trivial rerooting factor. Note that our derivation of Proposition 3 from Proposition 2 requires at least two faces $(F \geqslant 2)$ but formula (3.5) is also correct at $F=1\left(q_{j}=0\right.$ for all $j$, hence $\left.E=m\right)$, where the r.h.s. yields the $m$-th Catalan number, as wanted.

For instance, we may obtain the number of rooted $2 m$-angulations $(m \geqslant 2)$ without multiple edges, with $F$ faces, by simply setting $q_{j}=(F-1) \delta_{j, m}$, namely

$$
2 m\left(\begin{array}{c}
2 m-1 \\
m+1
\end{array}\right) \frac{1}{(F-1) !}\left(\begin{array}{c}
2 m-1 \\
m+1
\end{array}\right)^{F-1} \frac{(E+F-3) !}{E !}=2 m\left(\begin{array}{c}
2 m-1 \\
m+1
\end{array}\right)^{F} \frac{((m+1) F-3) !}{(F-1) !(m F) !}
$$

since $E=m F$ in this case. For $m=2$, this yields the well-known number of quadrangulations without multiple edges $[5,6,7,8,9]$

$$
4 \frac{(3 F-3) !}{(F-1) !(2 F) !}=2 \frac{(3 F-3) !}{F !(2 F-1) !}
$$

in terms of the number $F$ of faces. 


\subsection{4-irreducible maps}

Another case of interest concerns 4-irreducible maps, which requires the knowledge of $r^{(4)}(z)$. From (3.1), we immediately obtain

$$
r^{(4)}(z)=1+\frac{1-\sqrt{1-4 z}}{2} .
$$

After some straightforward algebra, we find, for $p>0$ and $s \geqslant 0$

$$
\begin{aligned}
\frac{s !}{p}\left[z^{s}\right]\left(r^{(4)}\right)^{p} & =\frac{\delta_{s, 0}}{p}+(s-1) ! \sum_{\ell=0}^{\min (p-1, s-1)}\left(\begin{array}{c}
p-1 \\
\ell
\end{array}\right)\left(\begin{array}{c}
2(s-1)-\ell \\
s-1
\end{array}\right) \\
& =\frac{\delta_{s, 0}}{p}+\frac{(2(s-1)) !}{(s-1) !}{ }_{2} F_{1}(1-p, 1-s, 2(1-s) ;-1)
\end{aligned}
$$

in terms of the hypergeometric function ${ }_{2} F_{1}$ (here the second term in the r.h.s. must be understood as 0 if $s=0)$. Using this expression at $p=E+2 F-4-4 q_{2}$ and $s=F-2$, we arrive at the formula

$$
\begin{aligned}
N_{m}^{(4)}\left(\left\{q_{j}\right\}_{j \geqslant 2}\right)= & (2 m)\left(\begin{array}{c}
2 m-1 \\
m+2
\end{array}\right) \prod_{j>2} \frac{1}{q_{j} !}\left(\begin{array}{c}
2 j-1 \\
j+2
\end{array}\right)^{q_{j}} \\
& \times\left\{\frac{\delta_{F, 2}}{E}+\frac{(2(F-3)) !}{q_{2} !(F-3) !}{ }_{2} F_{1}\left(5-E-2 F+4 q_{2}, 3-F, 2(3-F) ;-1\right)\right\},
\end{aligned}
$$

valid for $F \geqslant 2+q_{2}$, i.e. with at least two faces of degree strictly larger than 4 (note that setting $F=2$ implies necessarily $q_{2}=0$ ). As before, the second term is understood as 0 if $F=2$.

A simple application concerns maps with exactly two faces of degree strictly larger than 4: the root face of degree $2 m(m>2)$ and another face of degree $2 m^{\prime}\left(m^{\prime}>2\right)$. We have in this case $q_{j}=\delta_{j, m^{\prime}}+q_{2} \delta_{j, 2}, E=m+m^{\prime}+2 q_{2}$ and $F=2+q_{2}$, so that we find a number of rooted 4 -irreducible maps equal two

$$
2 m\left(\begin{array}{c}
2 m-1 \\
m+2
\end{array}\right)\left(\begin{array}{c}
2 m^{\prime}-1 \\
m^{\prime}+2
\end{array}\right)\left\{\frac{\delta_{q_{2}, 0}}{m+m^{\prime}}+\frac{\left(2\left(q_{2}-1\right)\right) !}{q_{2} !\left(q_{2}-1\right) !}{ }_{2} F_{1}\left(1-\left(m+m^{\prime}\right), 1-q_{2}, 2\left(1-q_{2}\right),-1\right)\right\}
$$

where the second term is to be understood as 0 for $q_{2}=0$. For $m=m^{\prime}=3$, it gives the sequence

$$
1,6,21,62,180,540,1683,5418,17901,60390,207207,720954,2537964,9023328
$$

whose first three terms correspond to the maps displayed in fig. 7 .

\subsection{Maps with girth at least 6}

If we specialize the result of the previous subsection to the case $q_{2}=0$, we obtain the number of rooted maps with girth at least 6 , with outer degree $2 m(m \geqslant 3)$ and with $q_{j}$ 

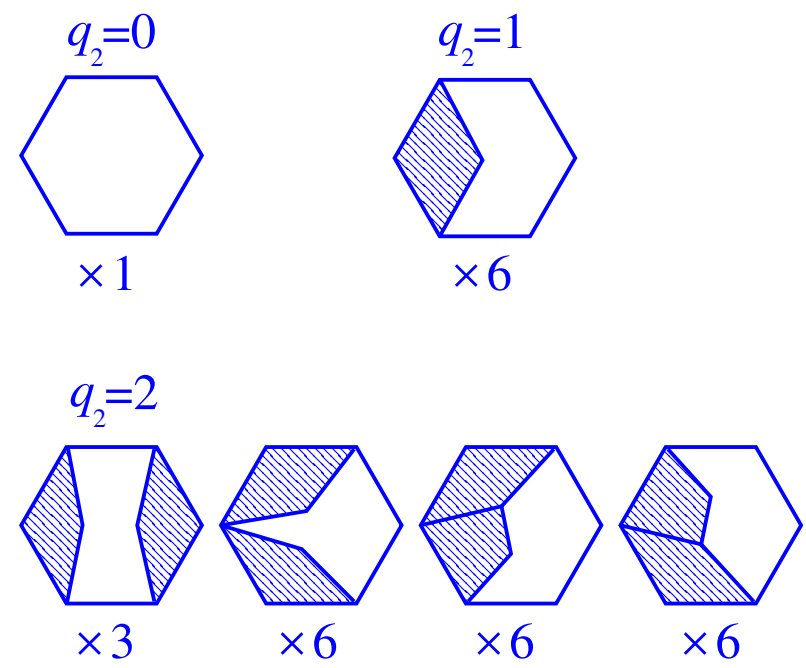

Figure 7: 4-irreducible maps made of two hexagons and $q_{2}$ squares, for $q_{2}=0,1$ and 2 . For each map, we indicated its multiplicity corresponding to the number of inequivalent possible choices of a root edge (not drawn here) incident to the outer hexagonal face.

non-root faces of degree $2 j(j \geqslant 3)$ :

$$
\begin{aligned}
\mathcal{N}_{m}^{(6)}\left(\left\{q_{j}\right\}_{j \geqslant 3}\right)= & (2 m)\left(\begin{array}{c}
2 m-1 \\
m+2
\end{array}\right) \prod_{j>2} \frac{1}{q_{j} !}\left(\begin{array}{c}
2 j-1 \\
j+2
\end{array}\right)^{q_{j}} \\
& \times\left\{\frac{\delta_{F, 2}}{E}+\frac{(2(F-3)) !}{(F-3) !}{ }_{2} F_{1}(5-E-2 F, 3-F, 2(3-F) ;-1)\right\} .
\end{aligned}
$$

If all faces have degree 6 , i.e. $m=3, q_{j}=(F-1) \delta_{j, 3}$, and $E=3 F$, we obtain the number of rooted hexangulations of girth 6 with $F$ faces

$$
\delta_{F, 2}+6 \frac{(2(F-3)) !}{(F-1) !(F-3) !}{ }_{2} F_{1}(5(1-F), 3-F, 2(3-F) ;-1)
$$

(as before, the second term is to be understood as 0 when $F=2$ ). It gives the sequence

$$
1,3,17,128,1131,11070,116317,1287480,14829188,176250143,2148687567
$$

corroborating the result of $[3,10]$.

\section{The case $b=0$}

It is interesting to include in our framework the case of general bipartite maps, without constraints of irreducibility. Indeed, we observe that, while our derivation of Theorem 1 and its consequences was done assuming $b \geqslant 1$, previously known formulas for general bipartite maps may be recovered by taking formally $b=0$. This should not come as a 


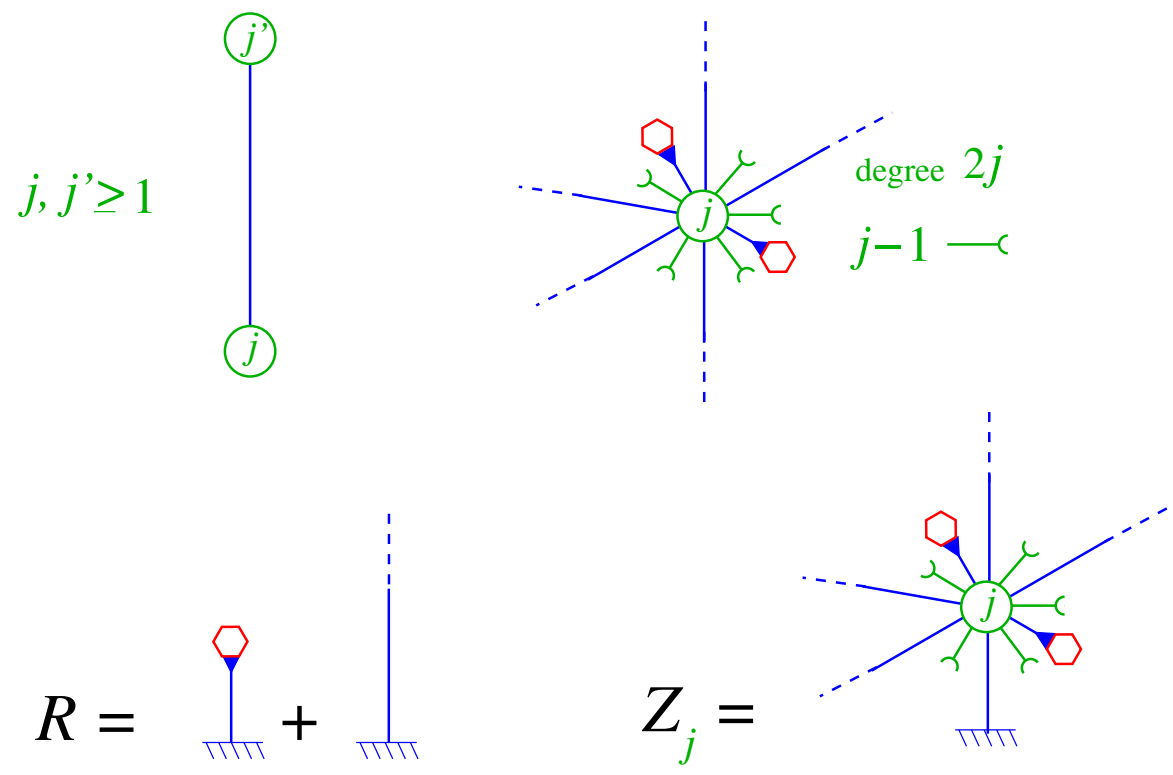

Figure 8: Building rules of the trees enumerated by $R$ and $Z_{j}$ satisfying eq. (4.2).

surprise since general bipartite maps correspond indeed to 0-irreducible bipartite maps. Eq. (2.1) at $b=0$ matches exactly the known formula [2]

$$
F_{2 j_{1}, 2 j_{2}}=2 j_{1}\left(\begin{array}{c}
2 j_{1}-1 \\
j_{1}
\end{array}\right) 2 j_{2}\left(\begin{array}{c}
2 j_{2}-1 \\
j_{2}
\end{array}\right) \frac{R^{j_{1}+j_{2}}}{j_{1}+j_{2}} \quad j_{1}, j_{2}>b
$$

for the generating function of bipartite maps with two boundaries of length $2 j_{1}$ and $2 j_{2}$, if we take as $R^{(0)}$ the solution $R$ of

$$
R=z+\sum_{j \geqslant 1} Z_{j}, \quad Z_{j}=\left(\begin{array}{c}
2 j-1 \\
j
\end{array}\right) x_{2 j} R^{j}
$$

which replaces the system (2.2)-(2.4) whenever $b=0$. Here $z$ corresponds to a weight per vertex of the map, which again is not surprising since, in some sense, we may view the vertices as faces of degree 0 .

The associated trees obey the rules displayed in fig. 8, and are nothing but the wellknown "blossom trees" introduced in $[11,12]$, with "buds" and "leaves" corresponding respectively to our buds and our blossoms enhanced by a leaf-vertex. Setting $b=0$ in (1.3) yields the known beautiful formula [2]

$$
F_{2 j_{1}, 2 j_{2}, \ldots, 2 j_{r}}=\frac{1}{\sum_{\ell=1}^{r} j_{\ell}} \prod_{\ell=1}^{r} 2 j_{\ell}\left(\begin{array}{c}
2 j_{\ell}-1 \\
j_{\ell}
\end{array}\right) \frac{\partial^{r-2}}{\partial z^{r-2}} R_{\ell=1}^{\sum_{\ell=1}^{r} j_{\ell}}
$$

for the generating function of bipartite maps with multiple boundaries. Taking $r^{(0)}=z$ and formally $q_{0}=V$ and $F=2+E$ (indeed $F$ must be understood here as the number of 
real faces plus that of degree- 0 faces, i.e. vertices, a sum which equals $2+E$ from Euler's relation), eq. (3.2) yields

$$
N_{m}\left(\left\{q_{j}\right\}_{j \geqslant 1}\right)=(2 m)\left(\begin{array}{c}
2 m-1 \\
m
\end{array}\right) \prod_{j \geqslant 1} \frac{1}{q_{j} !}\left(\begin{array}{c}
2 j-1 \\
j
\end{array}\right)^{q_{j}} \times \frac{(E-1) !}{V !}
$$

which is the well-known Tutte's formula for the number $N_{m}\left(\left\{q_{j}\right\}_{j \geqslant 1}\right)$ of bipartite maps with root face of degree $2 m$ and with $q_{j}$ (non-root) faces of degree $2 j$ [13]. Here $E=$ $m+\sum_{j \geqslant 1} j q_{j}$ and $V=m+1+\sum_{j \geqslant 1}(j-1) q_{j}$ denote the number of edges and vertices respectively.

\section{Concluding remarks}

Upon derivation with respect to $z$, formula (1.3) yields

$$
\frac{\partial}{\partial z} F_{2 j_{1}, 2 j_{2}, \ldots, 2 j_{r}}^{(d)}=\frac{1}{(r-2) b+\sum_{\ell=1}^{r} j_{\ell}} \prod_{\ell=1}^{r} 2 j_{\ell}\left(\begin{array}{c}
2 j_{\ell}-1 \\
j_{\ell}+b
\end{array}\right) \frac{\partial^{r-1}}{\partial z^{r-1}}\left(R^{(d)}\right)^{(r-2) b+\sum_{\ell=1}^{r} j_{\ell}}
$$

In this form, it remains valid at $r=1$ since, as shown in [1], the generating function for $d$-irreducible maps with a single boundary of length $2 j_{1}$ satisfies the so-called "pointing formula"

$$
\frac{\partial F_{2 j_{1}}^{(d)}}{\partial z}=\left(\begin{array}{c}
2 j_{1} \\
j_{1}-b
\end{array}\right)\left(R^{(d)}\right)^{j_{1}-b}=\frac{2 j_{1}}{-b+j_{1}}\left(\begin{array}{c}
2 j_{1}-1 \\
j_{1}+b
\end{array}\right)\left(R^{(d)}\right)^{-b+j_{1}} \quad j_{1}>b .
$$

Conversely, we may use eq. (5.2) as a starting point to derive eq. (5.1) from the identity

$$
F_{2 j_{1}, 2 j_{2}, \ldots, 2 j_{r}}^{(d)}=\left(\prod_{\ell=2}^{r} 2 j_{\ell} \frac{\partial}{\partial x_{2 j_{\ell}}}\right) F_{2 j_{1}}^{(d)}, \quad r \geqslant 1
$$

by following the same procedure as above via a decomposition of the appropriate trees with marked white vertices into forests. Still going back from (5.1) to (1.3) is not a simple matter as it requires integrating over $z$ and gives rise to some a priori unknown integration constant (which is $z$-independent but depends on the $j_{\ell}$ 's). Fixing this constant requires knowing the value of $F_{2 j_{1}, 2 j_{2}, \ldots, 2 j_{r}}^{(d)}$ at some particular value of $z$ (for instance at $z=0$ where it enumerates maps with girth at least $d+2$ ), a problem whose difficulty might be comparable to that of the initial problem. This is why we chose instead the generating function of maps with two boundaries as starting point.

Finally, we would like to stress that we used here trees as a simple tool to evaluate the wanted map generating functions and did not recourse to any direct bijection between maps and trees. Nevertheless, we know from [1] that such direct bijections do exist between the trees enumerated by $R^{(d)}$ and $U_{k}^{(d)}, k \geqslant 0$ and the so-called slices or $k$-slices 
(which are particular instances of $d$-irreducible maps) enumerated by the same functions. Ref. [1] describes in details a direct correspondence between trees and slices in the case $x_{2 j}=0, j>b$, and this correspondence can easily be extended to the case of nonvanishing $x_{2 j}$ 's. To obtain a direct bijection between $d$-irreducible maps with several boundaries and trees, we would simply need a direct bijection between $d$-irreducible maps with two boundaries of lengths $2 j_{1}$ and $2 j_{2}$ and the trees enumerated by the r.h.s of (2.1). We have not found any such bijection so far. In particular, eq. (2.1) still awaits a direct bijective proof. This is to be contrasted with eq. (5.2), which was given in [1] a direct bijective proof, which may be reformulated as a direct bijection with trees. However, as we just discussed, this formula is not sufficient to recover our main result (1.3).

\section{Acknowledgements}

The work of JB was partly supported by the ANR projects "Cartaplus" 12-JS02-001-01 and "IComb" ANR-08-JCJC-0011.

\section{References}

[1] J. Bouttier and E. Guitter. On irreducible maps and slices. To appear in Combinatorics, Probability and Computing, special issue dedicated to the Memory of Philippe Flajolet, arXiv:1303.3728 [math.CO].

[2] G. Collet and É. Fusy. A simple formula for the series of bipartite and quasi-bipartite maps with boundaries. FPSAC 2012, Nagoya, Japan, DMTCS proc., AR:607-618, 2012, arXiv:1205.5215 [math.CO].

[3] O. Bernardi and É. Fusy. A bijection for triangulations, quadrangulations, pentagulations, etc. J. Combin. Theory Ser. A, 119:218-244, 2012, arXiv:1007.1292 [math.CO].

[4] O. Bernardi and É. Fusy. Unified bijections for maps with prescribed degrees and girth. J. Combin. Theory Ser. A, 119:1351-1387, 2012, arXiv:1102.3619 [math.CO].

[5] W.T. Tutte. A Census of planar maps. Canad. J. Math., 15:249-271, 1963.

[6] W.G. Brown. Enumeration of non-separable planar maps. Can. J. Math., 15:526-554, 1963.

[7] W.G. Brown and W.T. Tutte. On the enumeration of rooted non-separable planar maps. Canad. J. Math., 16:572-577, 1964.

[8] G. Schaeffer and B. Jacquard. A bijective census of non-separable planar maps. J. Comb. Theory A, 83:1-20, 1998.

[9] J. Bouttier and E. Guitter. Distance statistics in quadrangulations with no multiple edges and the geometry of minbus. J. Phys. A: Math. Theor., 43:205207, 2010, arXiv:1002.2552 [math-ph].

[10] see Sequence A179300 in The On-Line Encyclopedia of Integer Sequences, published electronically at http://oeis.org, 2010. 
[11] G. Schaeffer. Conjugaison d'arbres et cartes combinatoires aléatoires. PhD Thesis, Université Bordeaux I (1998).

[12] G. Schaeffer. Bijective census and random generation of Eulerian planar maps. Electron. J. Combin., 4:R20, 1997.

[13] W.T. Tutte. A Census of slicings. Canad. J. Math., 14:708:722, 1962. 